\title{
Multi-graviton theory from a discretized RS brane-world and the induced cosmological constant
}

\author{
Guido Cognola $^{1 a}$, Emilio Elizalde ${ }^{2 b}$, Shin'ichi Nojiri ${ }^{3 c}$, \\ Sergei D. Odintsov ${ }^{4 d}$ and Sergio Zerbini ${ }^{5 a}$ \\ ${ }^{a}$ Dipartimento di Fisica, Università di Trento \\ and Istituto Nazionale di Fisica Nucleare, \\ Gruppo Collegato di Trento, Italia \\ ${ }^{b}$ Consejo Superior de Investigaciones Científicas \\ IEEC, Edifici Nexus 201, Gran Capità 2-4, 08034 Barcelona, Spain, \\ and Departament ECM and IFAE, Facultat de Física, \\ Universitat de Barcelona, Diagonal 647, 08028 Barcelona, Spain \\ ${ }^{c}$ Department of Applied Physics, National Defence Academy, \\ Hashirimizu Yokosuka 239-8686, JAPAN \\ ${ }^{d}$ Institut d'Estudis Espacials de Catalunya (IEEC), \\ Edifici Nexus, Gran Capità 2-4, 08034 Barcelona, Spain, and \\ Instituciò Catalana de Recerca i Estudis Avançats (ICREA), Barcelona, Spain
}

\begin{abstract}
We propose a multi-graviton theory with non-nearest-neighbor couplings in the theory space. The resulting four-dimensional discrete mass spectrum reflects the structure of a latticized extra dimension. For a plausible mass spectrum motivated by the discretized Randall-Sundrum brane-world, the induced cosmological constant turns out to be positive and may serve as a quite simple model for the dark energy of our accelerating universe.
\end{abstract}

\footnotetext{
${ }^{1} \mathrm{e}-\mathrm{mail}$ : cognola@science.unitn.it

2e-mail: elizalde@ieec.fcr.es, elizalde@math.mit.edu

${ }^{3}$ e-mail: nojiri@nda.ac.jp, snojiri@yukawa.kyoto-u.ac.jp

${ }^{4}$ e-mail: odintsov@ieec.fcr.es Also at TSPU, Tomsk, Russia

${ }^{5}$ e-mail: zerbini@science.unitn.it
} 
1. Introduction. There is renewed interest in the study of multi-graviton theories [1]. Generally speaking, the corresponding action can be viewed as a sum of Pauli-Fierz terms while a consistent interaction of gravitons does not occur there. In a way, multigraviton theories resemble higher-dimensional gravities with a discretized dimension. And such sort of discretized Kaluza-Klein theories are now under intense attention due to their primary importance in the realization of the dimensional deconstruction program $[2,3]$. Moreover, multi-gravitons are also related with discretized brane-worlds [4].

In spite of the the absence of a consistent interaction among the gravitons, one can think on possible couplings in the theory space. In particular, in a recent paper [5] a multi-graviton theory with nearest-neighbour couplings in the theory space has been proposed. As a result, a discrete mass spectrum appears. The theory seems to be equivalent to Kaluza-Klein gravity with a discretized dimension.

In this letter, we propose a simple model of a multi-graviton theory with nonnearest-neighbour couplings in the theory space. Depending on the choice for such couplings, a the different graviton mass spectrum is induced. For instance, our model may be seen as motivated by a discretized Randall-Sundrum (RS) brane-world [6] or by complicated boundary condition on a latticized dimension. It opens number of possibilities for the choice of mass spectrum. For example motivated by discretized RS brane-world model the small positive cosmological constant is induced as shown by the explicit one-loop calculation.

2. A multi-graviton model. We consider $N$ variables, $\phi_{n}$, which may be identified with the fields on a lattice with $N$ sites. The difference operator $\Delta$ is defined by

$$
\Delta \phi_{n} \equiv \sum_{k=0}^{N-1} a_{k} \phi_{n+k}
$$

Here we assume $\phi_{n+N}=\phi_{n}$, which may be regarded as a periodic boundary condition. In (1), the $a_{k}$ 's are $N$ constants. In order that $\Delta$ becomes a usual differentiation in a proper continuum limit, the following condition is imposed:

$$
\sum_{k=0}^{N-1} a_{k}=0
$$

The eigenvectors for $\Delta$ are given by

$$
\phi_{n}^{M}=\frac{1}{\sqrt{N}} \mathrm{e}^{i \frac{2 \pi n M}{N}}, \quad M=0,1,2,3
$$

and their corresponding eigenvalues, by

$$
\Delta \phi_{n}^{M}=i m^{M} \phi_{n}^{M}, \quad i m^{M}=\sum_{n=0}^{N-1} a_{n} \mathrm{e}^{i \frac{2 \pi n M}{N}} .
$$

Here $m^{0}=0$. We should also note that $\phi_{n}^{M}$ satisfies the following properties, which may be identified with the conditions of normalization and completeness, respectively:

$$
\sum_{n=0}^{N-1} \phi_{n}^{M *} \phi_{n}^{M^{\prime}}=\delta^{M M^{\prime}}, \quad \sum_{M=0}^{N-1} \phi_{n}^{M *} \phi_{n^{\prime}}^{M}=\delta_{n n^{\prime}} .
$$


One can solve $a_{n}$ with respect to $m^{M}$ by

$$
a_{n}=\frac{i}{\sqrt{N}} \sum_{M=0}^{N-1} m^{M} \phi_{n}^{M *}=\frac{i}{N} \sum_{M=0}^{N-1} m^{M} \mathrm{e}^{-i \frac{2 \pi n M}{N}} .
$$

Then by choosing $a_{n}$ properly, we may obtain arbitrary spectrum of $m^{M}$ with $m^{0}=0$. We should note if $a_{n}$ is real

$$
m^{N-M}=-\left(m^{M}\right)^{*} .
$$

In what follows, we will assume both that $a_{n}$ is real and that the condition (7) is satisfied. For instance, if $N=2$, we find that $m^{1}$ is pure imaginary and that

$$
a_{0}=-\Im m^{1}, a_{1}=\Im m^{1} \text {. }
$$

Here $\Im m^{1}$ is the imaginary part of $m^{1}$, and we denote the real part of $m$ by $\Re m$. For $N=3$, we have $m^{2}=-\left(m^{1}\right)^{*}$, and

$$
\begin{aligned}
& a_{0}=-\frac{2}{3} \Im m^{1}, \quad a_{1}=\frac{1}{3}\left(\Im m^{1}+\sqrt{3} \Re m_{1}\right), \\
& a_{2}=\frac{1}{3}\left(\Im m^{1}-\sqrt{3} \Re m_{1}\right) .
\end{aligned}
$$

For $N=4$, we have $m^{3}=-\left(m^{1}\right)^{*}$ and find $m^{2}$ is pure imaginary. Then we obtain

$$
\begin{aligned}
a_{0}=\frac{1}{4}\left(2 \Im m^{1}-\Im m^{2}\right), & a_{1}=\frac{1}{4}\left(2 \Re m^{1}+\Im m^{2}\right), \\
a_{2}=\frac{1}{4}\left(-2 \Im m^{1}-\Im m^{2}\right), & a_{3}=\frac{1}{4}\left(-2 \Re m^{1}+\Im m^{2}\right) .
\end{aligned}
$$

One may apply the above arguments to the multi-graviton theory, extending the formalism in [5].

The Lagrangian of the massless spin-two field (graviton) $h_{\mu \nu}$ is given by

$$
\mathcal{L}_{0}=-\frac{1}{2} \partial_{\lambda} h_{\mu \nu} \partial^{\lambda} h^{\mu \nu}+\partial_{\lambda} h^{\lambda}{ }_{\mu} \partial_{\nu} h^{\mu \nu}-\partial_{\mu} h_{\mu \nu} \partial_{\nu} h+\frac{1}{2} \partial_{\lambda} h \partial^{\lambda} h,
$$

where $h \equiv h^{\mu}{ }_{\mu}$. The Lagrangian of the massive graviton with mass $m$ is given by introducing Stückerberg fields $A_{\mu}$ and $\varphi[7]$

$$
\begin{aligned}
\mathcal{L}_{m}= & \mathcal{L}_{0}-\frac{m^{2}}{2}\left(h_{\mu \nu} h^{\mu \nu}-h^{2}\right)-2\left(m A^{\mu}+\partial^{\mu} \varphi\right)\left(\partial^{\nu} h_{\mu \nu}-\partial h\right) \\
& -\frac{1}{2}\left(\partial_{\mu} A_{\nu}-\partial_{\nu} A_{\mu}\right)\left(\partial^{\mu} A^{\nu}-\partial^{\nu} A^{\mu}\right) .
\end{aligned}
$$

We consider $N$ copies of the graviton fields $h_{n \mu \nu}$ and Stückerberg fields $A_{n \mu}$ and $\varphi_{n}$. By replacing

$$
h_{\mu \nu} \rightarrow h_{n \mu \nu}, \quad A_{\mu} \rightarrow-i A_{n \mu}, \quad \varphi \rightarrow \varphi_{n}, \quad m \rightarrow-i \Delta,
$$

and adding all terms in the action ( $\operatorname{sum}$ on $n$ ), we hereby propose a theory given by the following Lagrangian, which is a generalization of the one in [5]:

$$
\begin{aligned}
\mathcal{L}= & \sum_{n=0}^{N-1}\left[-\frac{1}{2} \partial_{\lambda} h_{n \mu \nu} \partial^{\lambda} h_{n}^{\mu \nu}+\partial_{\lambda} h_{n \mu}^{\lambda} \partial_{\nu} h_{n}^{\mu \nu}-\partial_{\mu} h_{n \mu \nu} \partial_{\nu} h_{n}+\frac{1}{2} \partial_{\lambda} h_{n} \partial^{\lambda} h_{n}\right. \\
& +\frac{1}{2}\left(\Delta h_{n \mu \nu} \Delta h_{n}^{\mu \nu}-\left(\Delta h_{n}\right)^{2}\right)-2\left(\Delta^{\dagger} A_{n}^{\mu}+\partial^{\mu} \varphi_{n}\right)\left(\partial^{\nu} h_{n \mu \nu}-\partial_{\mu} h_{n}\right) \\
& \left.+\frac{1}{2}\left(\partial_{\mu} A_{n \nu}-\partial_{\nu} A_{n \mu}\right)\left(\partial^{\mu} A_{n}^{\nu}-\partial^{\nu} A_{n}^{\mu}\right)\right] .
\end{aligned}
$$


Here $\Delta$ is the difference operator, defined in (1), which operates on the indices $n$, while $\Delta^{\dagger}$ is defined as

$$
\Delta^{\dagger} \phi_{n} \equiv \sum_{k=0}^{N-1} a_{k} \phi_{n-k},
$$

which satisfies the following equation:

$$
\sum_{n=0}^{N-1} \phi_{n}^{1} \Delta \phi_{n}^{2}=\sum_{n=0}^{N-1}\left(\Delta^{\dagger} \phi_{n}^{1}\right) \phi_{n}^{2} .
$$

The Lagrangian is invariant under transformations with the local parameters $\xi_{n}^{\nu}$ and $\zeta_{n}$ :

$$
\begin{aligned}
h_{n \mu \nu} & \rightarrow h_{n \mu \nu}+\partial_{\mu} \xi_{n \nu}+\partial_{\nu} \xi_{n \mu} \\
A_{n \mu} & \rightarrow A_{n \mu}+\Delta \xi_{n \mu}-\partial_{\mu} \zeta_{n} \\
\varphi_{n} & \rightarrow \varphi_{n}+\Delta^{\dagger} \zeta_{n}
\end{aligned}
$$

Since the spectrum of $\Delta$ is given by (4), the Lagrangian describes a massless graviton (with mass $\left.m^{0}=0\right)$ and $N-1$ massive gravitons with masses $\left|m^{M}\right|(M=1,2, \cdots, N-$ 1) in (4). We should point out that the massive gravitons always appear in pairs, sharing a common mass. As we have shown, the complex mass parameter $m^{M}(M=$ $1,2, \cdots, N-1)$ can be arbitrary chosen, just by properly choosing the coefficients $a_{k}$ in (1) to (6). As discussed in [5], the Lagrangian (14) can be regarded as corresponding to a Kaluza-Klein theory where the extra dimension is a lattice.

As an example, we consider the two-brane RS model [6] (for a recent review, see [8]). In that model, the masses $m^{M}$ of the Kaluza-Klein modes are given by the solutions of the following equation:

$$
\begin{aligned}
0= & \left(y_{2}^{\prime}(0)-\frac{3}{2 l} y_{2}(0)\right)\left(j_{2}^{\prime}\left(z_{c}\right)+\frac{3}{2\left(l+z_{c}\right)} j_{2}\left(z_{c}\right)\right) \\
& -\left(j_{2}^{\prime}(0)-\frac{3}{2 l} j_{2}(0)\right)\left(y_{2}^{\prime}\left(z_{c}\right)+\frac{3}{2\left(l+z_{c}\right)} y_{2}\left(z_{c}\right)\right) .
\end{aligned}
$$

Here $l$ is the length parameter of the five-dimensional AdS space and $z_{c}$ is given by the geodesic distance $\pi r_{c}$ between the two branes as

$$
z_{c}=l\left(\mathrm{e}^{\frac{\pi r_{c}}{l}}-1\right)
$$

The functions $j_{2}(z)$ and $y_{2}(z)$ are given in terms of Bessel functions as

$$
j_{2}(z)=\sqrt{z+l} J_{2}\left(m^{M} z+l\right), \quad y_{2}(z)=\sqrt{z+l} Y_{2}\left(m^{M} z+l\right) .
$$

Specifically, we can consider models given by a first few solutions for $m^{M}$ of Eq. (18), in the different cases (8), (9), and (10). On the other hand, when $m^{M}$ is large, the Bessel functions behave as

$$
\begin{aligned}
& \sqrt{z} J_{2}\left(m^{M} z\right) \sim \sqrt{\frac{2}{\pi m^{M}}} \cos \left(m^{M} z-\frac{5}{4} \pi\right), \\
& \sqrt{z} Y_{2}\left(m^{M} z\right) \sim \sqrt{\frac{2}{\pi m^{M}}} \sin \left(m^{M} z-\frac{5}{4} \pi\right) .
\end{aligned}
$$


Then Eq. (18) reduces to

$$
\tan \left(m^{M} l-\frac{5}{2} \pi\right) \sim \tan \left(m^{M}\left(z_{c}+l\right)-\frac{5}{2} \pi\right),
$$

which has the solution

$$
m^{M}=\frac{\pi K}{z_{c}},
$$

$K$ being a positive integer. Motivated by (23), we may consider a $N=2 N^{\prime}+1$ graviton model with graviton masses given by

$$
m^{M}= \begin{cases}\frac{\pi M}{z_{c}}, & M=0,1, \cdots N^{\prime} \\ -\frac{\pi(N-M)}{z_{c}}, & M=N^{\prime}+1, N^{\prime}+2, \cdots N-1=2 N^{\prime} .\end{cases}
$$

Eq. (6) gives

$$
\begin{aligned}
a_{0} & =0 \\
a_{n} & =-\frac{2 \pi}{\left(2 N^{\prime}+1\right) z_{c}} \Im\left\{\frac{\left(1-\mathrm{e}^{-i \frac{2 \pi N^{\prime} n}{2 N^{\prime}+1}}\right) \mathrm{e}^{-i \frac{2 \pi n}{2 N^{\prime}+1}}}{1-\mathrm{e}^{-i \frac{2 \pi n}{2 N^{\prime}+1}}}\right\} \\
& =-\frac{(-1)^{n} 2 \pi}{N z_{c}} \frac{\sin ^{2}\left(\frac{\pi n N^{\prime}}{N}\right)}{\sin \left(\frac{\pi n}{N}\right)}, \quad(n \neq 0) .
\end{aligned}
$$

Then $N$ plays the role of a cutoff of the Kaluza-Klein modes.

In previous models $[3,5]$ of deconstruction, mainly nearest neighbour couplings between the sites of the lattice had been considered. Then, on imposing a periodic boundary condition, the lattice looks as a circle. Departing from this case, in the model considered here, we have introduced non-nearest-neighbour couplings among the sites. Then, a site links to a number of different ones in a rather complicated way. In this sense, the lattice in the present model is no more a simple circle but it looks like, say, a mesh or a net. Let us assume that the sites on the lattice correspond to points in a brane. If the codimension of the spacetime is one, the brane should be ordered, resembling the sheets of a book. One brane can only interact (directly) with the neighbouring two branes. However, if the spacetime is more complicated and/or the codimension is two or more, brane can (directly) interact with three or more branes, an interaction that will be perfectly described by our corresponding model. For example, a site on a tetrahedron connects with three neighbouring sites. In this way, the nonnearest couplings may adequately reflect the structure of the extra dimension. In this respect our model is very general and opens a number of interesting possibilities.

3. The induced cosmological constant. We will here compute the one-loop effective potential and as a consequence the induced cosmological constant for the $N$ graviton model discussed in the previous section (for a recent review on Casimir energy calculations from extra dimensions, see [9]). We shall use zeta-function regularization [10], but instead, a different regularization scheme could work as well. First of all we compute the effective potential for a free scalar field with mass $M$ since this corresponds to the contribution of each degree of freedom to the one-loop effective potential of our theory. 
In the zeta-function regularization scheme, the one-loop contribution to the effective potential is given by

$$
V_{e f f}^{(1)}=-\frac{1}{2 V} \zeta^{\prime}(0),
$$

$V$ being the volume of the manifold and $\zeta(s)$ the zeta function corresponding to the operator $L=-\nabla^{2}+M^{2}$. In a flat manifold it can be easily computed by means of

$$
\begin{aligned}
\zeta(s) & =\frac{1}{\Gamma(s)} \int_{0}^{\infty} d t t^{s-1} \operatorname{Tr} e^{-t L / \mu^{2}}=\frac{V \mu^{2 s}}{(4 \pi)^{d / 2} \Gamma(s)} \int_{0}^{\infty} d t t^{s-d / 2-1} e^{-t M^{2}} \\
& =\frac{V M^{d}}{(4 \pi)^{d / 2}} \frac{\Gamma(s-d / 2)}{\Gamma(s)}\left(\frac{M}{\mu}\right)^{-2 s}
\end{aligned}
$$

which is valid in arbitrary dimension $d$. The parameter $\mu$ has to be introduced for dimensional reasons and it will be fixed by renormalization. We should notice that the last expression provides the analytic continuation of the zeta function to the whole complex $s$-plane. One can see that in even dimensions there are a finite number of simple poles at the integer points $s=1,2, \ldots, d / 2$, while in odd dimensions there is an infinite number of simple poles at all the half-integer points $s \leq d / 2$. In particular, in 4 -dimensions the zeta function has only two simple poles, and reads

$$
\zeta(s)=\frac{V M^{4}}{32 \pi^{2}(s-1)(s-2)}\left(\frac{M}{\mu}\right)^{-2 s} .
$$

By taking the derivative of Eq. (27) with respect to $s$, we obtain

$$
V_{e f f}^{(1)}=\frac{(-1)^{d / 2} M^{d}}{2(d / 2) !(4 \pi)^{d / 2}}\left[\ln \frac{M^{2}}{\mu^{2}}-\alpha(d)\right],
$$

which is valid in even dimensions, and

$$
V_{e f f}^{(1)}=-\frac{M^{d} \Gamma(-d / 2)}{2(4 \pi)^{d / 2}}
$$

valid in odd dimensions. Here $\alpha(d)=1+1 / 2+1 / 3+\ldots+2 / d$ is a rational number larger than 1.

It has to be noted that, in the odd dimensional case, the effective potential does not depend on $\mu$ and, owing to the presence of the Euler's gamma function, it is alternatively negative and positive. In particular, it is positive in five dimensions. The situation drastically changes in even dimensions. In fact in such case there is an explicit dependence on the scale parameter, and the effective potential can be positive or negative, this depending on the value of $M / \mu$.

In the multi-graviton model which we are considering in this paper, the effective potential can be obtained by adding up several contributions of the kind (29) for $d=4$. The masses are given by Eq. (24). In our model we have a massless graviton (which does not give contributions to the effective potential) and an even number of massive gravitons ( with the same mass). Then, in order to perform the computation, it is sufficient to consider only one-half of the whole mass spectrum, that is

$$
M_{p}=\frac{\pi p}{z_{c}}, \quad \quad p=1,2, \ldots, \frac{N-1}{2} .
$$


In this way, we get

$$
\begin{aligned}
V_{e f f} & =V_{R}(\mu)+10 \sum_{p=1}^{\frac{N-1}{2}} \frac{M_{p}^{4}}{64 \pi^{2}}\left(\ln \frac{M_{p}^{2}}{\mu^{2}}-\frac{3}{2}\right) \\
& =V_{R}(\mu)+10 \sum_{p=1}^{\frac{N-1}{2}} \frac{\pi^{2} p^{4}}{64 z_{c}^{4}}\left(\ln \frac{\pi^{2} p^{2}}{z_{c}^{2} \mu^{2}}-\frac{3}{2}\right) \\
& =V_{R}(\mu)+\frac{2^{-10} N\left(N^{2}-1\right)\left(3 N^{2}-7\right) \pi^{2}}{3 z_{c}^{4}}\left(\ln \frac{\pi^{2}}{z_{c}^{2} \mu^{2}}-\frac{3}{2}\right) \\
& +10 \sum_{p=1}^{\frac{N-1}{2}} \frac{\pi^{2} p^{4}}{64 z_{c}^{4}} \ln p^{2},
\end{aligned}
$$

where $V_{R}(\mu)$ is the renormalized vacuum energy and the factor of 10 comes from the fact that there are two gravitons with the same mass, each of them having 5 scalar degrees of freedom.

The effective potential is a physical observable and for this reason it has not to depend on the choice of the arbitrary parameter scale $\mu$. This means that it has to satisfy to some renormalization condition (see e.g. [11])

$$
\mu \frac{d V_{e f f}}{d \mu}=0
$$

from which it follows

$$
V_{R}(\mu)=V_{R}\left(\mu_{R}\right)+\frac{2^{-10} N\left(N^{2}-1\right)\left(3 N^{2}-7\right) \pi^{2}}{3 z_{c}^{4}} \ln \frac{\mu^{2}}{\mu_{R}^{2}},
$$

$\mu_{R}$ being the renormalization point which can be fixed by the condition $V_{R}\left(\mu_{R}\right)=0$. In this way one finally gets

$$
\begin{gathered}
V_{e f f}=\frac{2^{-10} N\left(N^{2}-1\right)\left(3 N^{2}-7\right) \pi^{2}}{3 z_{c}^{4}}\left(\ln \frac{\pi^{2}}{z_{c}^{2} \mu_{R}^{2}}-\frac{3}{2}\right) \\
+10 \sum_{p=1}^{\frac{N-1}{2}} \frac{\pi^{2} p^{4}}{64 z_{c}^{4}} \ln p^{2} .
\end{gathered}
$$

It interesting to note that, whatever the value of $\mu_{R}$ is (in practice it is fixed by some standard renormalization condition), due to the particular form of the mass spectrum, the expression (35) turns out to be positive if the number of gravitons is sufficiently large. This is a quite natural way to obtain a positive cosmological constant. One can observe that for large values of $N$, the effective potential goes as const $\cdot N^{5}(\ln N) / z_{c}^{4}$.

Now let us consider (for comparison) the multigraviton model studied in Ref. [5]. Also in that case there is a massless graviton, which can be omitted, and $N-1$ gravitons with masses

$$
M_{p}=2 m \sin \left(\frac{\pi p}{N}\right), \quad p=1,2, \ldots, N-1 .
$$

$m$ being a positive constant. From (28) and (29) with $d=4$, it directly follows that

$$
\zeta(s)=\frac{m^{4}}{2 \pi^{2}(s-1)(s-2)}\left(\frac{2 m}{\mu}\right)^{-2 s} \sum_{p=1}^{N-1} \sin ^{4-2 s}\left(\frac{\pi p}{N}\right),
$$


and the corresponding effective potential, renormalized according to the procedure explained above, is

$$
\begin{aligned}
V_{\text {eff }} & =\frac{5 m^{4}}{4 \pi^{2}} \sum_{p=1}^{N-1} \sin ^{4}\left(\frac{\pi p}{N}\right)\left[\ln \frac{4 m^{2}}{\mu_{R}^{2}}-\frac{3}{2}+\ln \sin ^{2}\left(\frac{\pi p}{N}\right)\right] \\
& =\frac{15 N}{32 \pi^{2}}\left(\ln \frac{4 m^{2}}{\mu_{R}^{2}}-\frac{3}{2}\right)+\frac{5 m^{4}}{2 \pi^{2}} \sum_{p=1}^{N-1} \sin ^{4}\left(\frac{\pi p}{N}\right) \ln \sin \left(\frac{\pi p}{N}\right),
\end{aligned}
$$

where the factor 5 reflects again the scalar degrees of freedom of massive gravitons. The sign of the effective potential for this second example (Eq. (38)) can be positive or negative, depending on the value $m / \mu_{R}$, but, contrary to what happens in our model, it is always negative, for the renormalization mass $\mu_{R}$ is large as compared with $m$ (see [5]). The last expression has a nice behavior with $N$ as $N \rightarrow \infty$, namely $\sim \alpha N$, being $\alpha=-0.041180$.

We should now remark that these results correspond to our re-calculation of the model in [5]. In fact, we noticed that a zeta function with a strange behavior was obtained there: apparently the starting point was a standard representation for $\zeta(s)$, valid in 4 dimensions for $\Re s>2$, but after analytic continuation a representation for $\zeta(s)$ valid only for $\Re s<2$ was obtained (Eqs. (16) and (22) in [5]). This was probably because of an unclear treatment of the massless graviton, which gives a divergent contribution in the original expression. In particular, owing to the existence of this zero mode, it seems that the step from Eqs. (19) to (20) in [5] missed the fact that the contribution of $p=0$ is special: all integer values of $\ell$ (and not only those which are multiple of $N$ ) contribute. This further yields a correction to Eqs. (22) and (23) in [5], which is exactly of the same kind as the last term there but with $N=1$. Obviously, this new contribution is infinite for $q=1$ (see the last term of Eq. (23) in [5]). This is why our Eq. (38) differs from the corresponding one in [5]), what is rendered clear when comparing the large $N$ behavior of both.

4. Discussion. Let us now estimate the distance between the two branes in our multigraviton theory obtained from a discretized Randall-Sundrum model in order to induce the observable cosmological constant. We may write $V_{\text {eff }}$ as $V_{\text {eff }}=V_{0}\left(N, z_{c}, \mu\right) z_{c}^{-4}$; as the Hubble constant $H$ is $H \sim 10^{-33} \mathrm{eV}$ in the present universe, the effective cosmological constant, $\Lambda_{e f f}$, should be $\Lambda_{\text {eff }} \sim\left(10^{-33} \mathrm{eV}\right)^{2}$. Since the Planck scale is $10^{19} \mathrm{GeV} \sim 10^{28} \mathrm{eV}$, one gets $V_{\text {eff }} \sim \frac{\Lambda_{\text {eff }}}{\kappa^{2}} \sim 10^{-33 \times 2+28 \times 2}(\mathrm{eV})^{4}=10^{-10}(\mathrm{eV})^{4}$. Then if $V_{0}\left(N, z_{c}, \mu\right)$ is of order unity, we find that $z_{c} \sim 10^{2}$ to $10^{3} \mathrm{eV}$, and since the Planck length is $10^{-33} \mathrm{~cm}$, the value of this $z_{c}$ corresponds to $10^{-3}$ to $10^{-2} \mathrm{~cm}$. In the (continuum) model [12], if the number of extra dimensions is $n$, the length of the extra dimension will be $10^{\frac{30}{n}-17} \mathrm{~cm}$. Then, if $z_{c}$ corresponds to the length of the extra dimension, the value obtained corresponds to the $n=2$ case. We note that the value we have obtained for $z_{c}$ is within the present limits of the measurements.

As a consequence, the new multi-graviton theory we propose admits quite an arbitrary mass spectrum. To be more specific, a model of this kind may be induced by a discretized brane-world. The induced cosmological constant is then defined by quantum effects. With a reasonable choice for the distance between the branes, the induced cosmological constant lies actually within the range of the admissible observational values. Thus, a multi-graviton theory with non-nearest-neigbour couplings in the theory space can provide a useful model for dark energy in the current accelerating universe. 
The whole approach may open a new window in the realization of the deconstruction program for QED/QCD like theories. Indeed, by similar adjustments of the mass spectrum one is able to obtain quite noticeable changes in the four-dimensional scalar effective potential. And such a dependence of the effective potential on the choice of mass spectrum allows us directly to put bounds to reasonable latticized boundary conditions from phenomenologically accepted estimations of the four-dimensional scalar potential.

Acknowledgments This investigation has been supported by the Program INFN(Italy)-CICYT(Spain), by DGICYT (Spain), project BFM2000-0810 and by the Ministry of Education, Science, Sports and Culture of Japan under the grant n.13135208 (S.N.).

\section{References}

[1] N. Boulanger, T. Damour, L. Gualtieri and M. Henneaux, Nucl. Phys. B597 (2001) 127, hep-th/0007220; N. Boulanger, Fortsch. Phys. 50 (2002) 858, hepth/0111216.

[2] A. Sugamoto, Grav. Cosmol. 9 (2003) 91, hep-ph/0210235; M. Bander, Phys. Rev. D64 (2001) 105021; V. Jejjala, R. Leigh and D. Minic, Phys. Lett. B556 (2003) 71 , hep-th/0212057.

[3] N. Arkani-Hamed, A.G. Cohen and H. Georgi, Phys. Rev. Lett. 86 (2001) 47574761, hep-th/0104005; N. Arkani-Hamed, H. Georgi, M.D. Schwartz, Ann. Phys. NY 305 (2003) 96-118, hep-th/0210184; C.T. Hill, S. Pokorski and J. Wang, Phys. Rev. D64 (2001) 105005.

[4] T. Damour, I. Kogan and A. Papazoglou, Phys. Rev. D66 (2002) 104025, hepth/0206044; C. Deffayet and J. Mourad, Solutions of multigravity theories and discretized brane worlds, hep-th/0311125.

[5] N. Kan and K. Shiraishi, Class.Quant.Grav. 20 (2003) 4965, gr-qc/0212113.

[6] L. Randall and R. Sundrum, Phys. Rev. Lett. 83 (1999) 3370, hep-th/9905221.

[7] S. Hamamoto, Prog. Theor. Phys. 97 (1997) 327, hep-th/9611141.

[8] R. Maartens, Brane-world gravity, gr-qc/0312059.

[9] K. Milton, Grav. Cosmol. 8 (2002) 65.

[10] E. Elizalde, S. D. Odintsov, A. Romeo, A.A. Bytsenko and S. Zerbini, Zeta Regularisation Techniques with Applications. (World Scientific, Singapore, 1994); E. Elizalde, Ten physical applications of spectral zeta functions (Springer, Berlin, 1995); A.A. Bytsenko, G. Cognola, L. Vanzo and S. Zerbini. Phys. Rept. 266 (1996) 1.

[11] I.O. Cherednikov, Acta Phys. Slov. 52 (2002) 221, hep-th/0206245.

[12] N. Arkani-Hamed, S. Dimopoulos and G.R. Dvali, Phys.Lett. B429 (1998) 263272, hep-ph/9803315. 